\title{
Predictors of duloxetine adherence and persistence in patients with fibromyalgia
}

This article was published in the following Dove Press journal:

Journal of Pain Research

21 June 2012

Number of times this article has been viewed

\section{Zhanglin Cui \\ Yang Zhao \\ Diego Novick \\ Douglas Faries}

Eli Lilly and Company, Indianapolis, IN, USA
Correspondence: Zhanglin Cui Eli Lilly and Company, Lilly Corporate Center, DC 1079, Indianapolis, IN 46285, USA

Tel + I $31765 \mid 5935$

Fax + I 3172776097

Email cui_zhanglin@lilly.com
Objectives: Adherence to medication for the treatment of fibromyalgia (FM) is predictive of lower overall health-care costs, and thus a lower burden on both patients and providers. The objectives of this study were to examine the predictors of adherence to and persistence with duloxetine therapy among commercially insured FM patients, and to identify subgroups of patients with high duloxetine persistence and adherence.

Study design: This cross-sectional, retrospective study analyzed medical and pharmacy records over 1 year for patients in the US aged 18-64 years with FM who initiated (no prior 90-day use) duloxetine treatment in 2008.

Methods: Adherence to duloxetine was measured by medication possession ratio (MPR), with high adherence defined as MPR $\geq 0.8$. Persistence was defined as the duration of therapy from the index date to the earliest of: the ending date of the last prescription, the date of the first gap of $>15$ days between prescriptions, or the end of the study period (12 months). Demographic and clinical predictors of adherence were examined via multiple logistic regression (MLR), and subgroups of duloxetine-persistent and -adherent patients were identified using classification and regression trees (CART).

Results: Among 4660 duloxetine patients, 33\% achieved high adherence. Factors associated with high adherence from MLR included older age, North Central and Northeast regions, prior venlafaxine, pregabalin, selective serotonin reuptake inhibitor (SSRI), or other antidepressant use, or comorbid dyslipidemia or osteoarthritis (all $P<0.05$ ). CART analysis revealed that patients with prior antidepressant use, aged $\geq 46$, or prior osteoarthritis had higher MPR (all $P<0.05$ ), and patients aged $\geq 45$ with a history of SSRI, venlafaxine, or anticonvulsant use had longer duration of therapy (all $P<0.05$ ).

Conclusions: Patients with high adherence to and persistence with duloxetine were significantly older and had prior antidepressant use.

Keywords: duloxetine, medication adherence, medication persistence, subgroup analysis, fibromyalgia

\section{Introduction}

Fibromyalgia (FM) is a chronic and often debilitating syndrome characterized by chronic widespread musculoskeletal pain and stiffness, nonrestorative sleep, and fatigue. ${ }^{1}$ Given its many and diverse symptoms, some of which may present with other disorders, FM can be difficult to diagnose. ${ }^{2}$ Common comorbidities in FM patients include gastrointestinal disorders, migraines, respiratory or circulatory conditions, and mental and mood disorders. ${ }^{3}$ In the United States, the prevalence of FM is estimated at $2 \%-4 \%$, predominantly in women, and it increases with age. ${ }^{4}$ After osteoarthritis, 
FM represents the second most common disorder treated by rheumatologists. ${ }^{5}$ It is estimated that FM treatment translates into an estimated economic burden in excess of US $\$ 20$ billion annually. ${ }^{6}$

The predominant theory underlying the pathophysiology responsible for the increased pain sensitivity (ie, allodynia and hyperalgesia) in those with FM is central sensitization due to dysregulation of pain pathways. ${ }^{7}$ Both serotonin (5-HT) and norepinephrine (NE) are key modulators of endogenous pain inhibitory mechanisms via the descending pain inhibitory pathways in the brain and spinal cord. ${ }^{8}$ It has been postulated that in FM, the net inhibitory effect of critical monoamines, such as 5-HT and NE, is reduced or lost, consequently shifting the descending pain modulatory system from a state of inhibition toward a state of pain facilitation. ${ }^{9,10}$ This idea is supported by the clinical observation that FM patients have lower levels of 5-HT and NE metabolites in their cerebrospinal fluid compared to controls. ${ }^{11,12}$

Duloxetine is a potent and selective dual 5-HT and NE reuptake inhibitor that has been shown to be effective in treating patients with FM. ${ }^{13-16}$ As a consequence, duloxetine was approved in the US for the treatment of FM in 2008. Given its recent approval, there has not been ample opportunity to study the real-world use of duloxetine in managing FM. In this vein, only recently have observational studies been published examining adherence to duloxetine in FM patients. ${ }^{17-20}$ Previous studies have shown that FM patients on higher doses (ie, $>30 \mathrm{mg} /$ day) of duloxetine were more likely to adhere to treatment and that higher adherence was associated with lower commercial health-care costs. ${ }^{19,20}$

Given the importance of medication adherence and persistence to treatment outcomes, including costs, the present study was designed to examine the predictors of adherence to and persistence with duloxetine therapy among commercially insured FM patients who initiated duloxetine in 2008. Although previous reports have focused on adherence to duloxetine treatment and its predictors, ${ }^{17-20}$ we are unaware of any study that has examined either duloxetine persistence or its predictors in FM patients. A second study goal was to identify subgroups of FM patients who were persistent with or adherent to duloxetine using classification and regression tree (CART) analysis.

\section{Methods}

\section{Study design}

This was a cross-sectional, retrospective claims database analysis assessing 1-year adherence and persistence to duloxetine.

\section{Data sources}

The study sample was constructed from the Thompson Reuters MarketScan Commercial Claims and Encounters Database (January 1, 2007 to December 31, 2009). A summary of how the study sample was generated is listed in Table 1. The MarketScan Database is a de-identified, nationwide medical claims database licensed by Thompson Medstat (Ann Arbor, MI), which is compliant with the Health Insurance Portability and Accountability Act. The MarketScan Database includes insurance claims of inpatient, outpatient, emergency room, pharmacy, behavioral healthcare, and enrollment data for employees and their dependents from large employer-sponsored health insurance in the US. Historically, MarketScan databases have $>500$ million claim records, and the databases have claims data from approximately 100 payers annually. All records are linked via unique patient identifiers, which allow for comprehensive tracking of an individual's health-care utilization. Demographic and health insurance plan characteristics including age, gender, geographic region, health plan type, and enrollment status are available. Detailed health-care records included dates of service, provider type, payments, and International Classification of Diseases, Ninth Revision, Clinical Modification

Table I Cohort selection

\begin{tabular}{|c|c|c|}
\hline Step & Criteria & No of patients \\
\hline $\mathrm{I}$ & Thompson Reuters MarketScan Commercial Claims and Encounters Database (2008) & 606,857 \\
\hline 2 & $\begin{array}{l}\text { Patients initiated on duloxetine therapy from January I, } 2008 \text { to 3I December, } 2008 \text { with no record of duloxetine } \\
\text { use over the prior } 90 \text { days }\end{array}$ & 30,845 \\
\hline 3 & Age between 18 and 64 years as of the index date & 30,510 \\
\hline 4 & Continuous enrollment for 12 months prior to and 12 months following the index date & $1 \mathrm{I}, \mathrm{I} 80$ \\
\hline 5 & $\begin{array}{l}\text { At least one medical claim with an associated inpatient or outpatient diagnosis for fibromyalgia (ICD-9-CM 729.Ix) } \\
\text { during the I2-month pre-index period OR I month post-index period }\end{array}$ & 6794 \\
\hline 6 & At least 30 days ( $\geq 30$ days) cumulative duloxetine supply over the 12 -month post-index period & 6692 \\
\hline 7 & $\begin{array}{l}\text { Duloxetine patients with a diagnosis of diabetic peripheral neuropathic pain (ICD-9-CM: } 250.6 x \text { or } 357.2) \text { or } \\
\text { depression (ICD-9-CM: } 296.2 x, 296.3 x, 300.4 x, 309.1 x \text {, or } 31 \text { I.xx) in the prior } 12 \text { months were excluded }\end{array}$ & 4660 \\
\hline
\end{tabular}

Abbreviation: ICD-9-CM, International Classification of Diseases, Ninth Revision, Clinical Modification. 
(ICD-9-CM) diagnosis and procedure codes. Pharmacy claim files contain National Drug Code, dispense date, quantity dispensed, and plan and patient costs.

\section{Study procedures}

The study cohort selection criteria are summarized in Table 1. As shown, the target population of this study was adult FM patients aged 18-64 years who were initiated on duloxetine therapy. The inclusion criteria for this study were as follows. (1) Patients were initiated on duloxetine therapy between January 1, 2008 and December 31, 2008. The first filled duloxetine prescription was defined as the index date.

(2) Patients had no record of use of the study medication in the prior 90 days. (3) Patients had at least one medical claim with an associated inpatient or outpatient FM diagnosis (as defined using ICD-9-CM: 729.1x). The diagnosis had to occur from 1 year prior to the index date to 1 month after the index date. (4) Patients had continuous enrollment from 12 months prior to the index date to 12 months after the index date. (5) Patients were in the age range of 18-64 years as of the index date. (6) Patients had at least 30 days cumulative duloxetine supply over the 12-month period following the index date. Duloxetine patients with a diagnosis of diabetic peripheral neuropathic pain (ICD-9-CM: 250.6x or 357.2) or depression (ICD-9-CM: 296.2x, 296.3x, 300.4x, 309.1x, or $311 . x x)$ in the prior 12 months were excluded.

\section{Measures of adherence and persistence}

Patient adherence to duloxetine was reported based on medication possession ratio (MPR). The MPR was defined as the number of days that duloxetine was supplied from all claims during the 1-year study period after the index date divided by 365 days. High and low adherence to duloxetine were defined as MPRs of $\geq 0.80$ and $<0.80$, respectively. ${ }^{21}$ Thus a patient who filled prescriptions adequate to supply the drug for at least $80 \%$ (292 days) of the 1-year period was considered to have high adherence.

Patient persistence was measured using duration of therapy, defined as the duration of therapy from the index date to the earliest of either (1) the ending date of the last prescription; (2) the date of the first gap of $>15$ days between prescriptions; or (3) the end of the study period (12 months). ${ }^{21}$ High and low persistence with duloxetine were defined as durations of $\geq 180$ and $<180$ days, respectively. ${ }^{22}$

\section{Statistical analysis}

Patients were classified into two cohorts based on high or low adherence. Differences in demographic and clinical characteristics between the two adherence cohorts were examined via chi-square test (categorical variables) or $t$-test (continuous variables). A stepwise multiple logistic regression (MLR) analysis with 0.1 as the entry and 0.05 as the exit significance levels was used to determine predictors of adherence, adjusting for the following patient and prescription characteristics: patient age; gender; geographical region (Northeast, North Central, South, and West); health plan type (consumer-driven health plans; comprehensive, exclusive provider organization; health maintenance organization; high-deductible health plan; point of service; preferred provider organization; and other plans); comorbidities in the prior year; select psychiatric, chronic pain, and sleep disorders; and use of medications in the prior year (psychotropic, sleep, and pain).

In addition to comparisons between the dichotomous highand low-adherence and -persistence cohorts, factors associated with the best adherence and persistence outcomes were independently identified using CART analysis. Patients with FM were recursively partitioned into more homogeneous subgroups with respect to adherence or persistence based on the optimal factors (ie, demographic characteristics, comorbidities, or prior medications) at each split. Partitioning was stopped when adherence or persistence for the two subgroups resulting from a partition was not statistically different using a $\chi^{2}$ test (for binary adherence) or F-test (for MPR or duration of therapy) ( $\alpha=0.05)$ or the node size was less than 250 patients (about $5 \%$ of the whole population).

Analyses were conducted using SAS version 9.1 (SAS, Cary, NC) or Enterprise Miner version 5.2 (SAS), and the significance level was set a priori at $P<0.05$.

\section{Results \\ Demographic and clinical characteristics}

Of the final sample of 4660 patients, approximately $88.6 \%$ were female ( $n=4130$, vs 530 males), and average age was 48 years. Almost $77 \%$ of the patients lived in the North Central or Southern regions of the US, and $61 \%$ were enrolled in a preferred provider organization (Table 2).

Based on MPR, the "low" adherence cohort (MPR < 0.80) comprised 3122 of the 4660 patients $(67.0 \%)$, while the "high" adherence cohort $(\mathrm{MPR} \geq 0.80)$ comprised 1538 patients (33.0\%) (Table 2). The average MPRs for the low and high duloxetine-adherence cohorts were 0.33 and 0.94 , respectively. Regarding duloxetine persistence, the low-adherence cohort had an average duration of therapy of 76.3 days, whereas the high-adherence cohort had an average duration of therapy of 279.4 days. Overall, the most prevalent comorbid 
Table 2 Demographic characteristics among patients with fibromyalgia grouped by adherence to duloxetine over prior year

\begin{tabular}{|c|c|c|c|c|}
\hline & Overall & $\begin{array}{l}\text { Low adherence } \\
(M P R<0.80)\end{array}$ & $\begin{array}{l}\text { High adherence } \\
(M P R \geq 0.80)\end{array}$ & $\begin{array}{l}P \text { values }\left(\chi^{2} \text { or }\right. \\
t \text { statistics) }\end{array}$ \\
\hline Number of patients, $n(\%)$ & $4660(100.0)$ & $3122(67.0)$ & $1538(33.0)$ & \\
\hline Duration of therapy, days & 143.3 & 76.3 & 279.4 & \\
\hline MPR & 0.53 & 0.33 & 0.94 & \\
\hline Gender, n (\%) & & & & $0.70(0.15)$ \\
\hline Male & $530(I I .4)$ & $359(11.5)$ & $17 \mid(\mid 1.1)$ & \\
\hline Female & $4130(88.6)$ & $2763(88.5)$ & I $367(88.9)$ & \\
\hline Age, years, n (\%) & & & & $<0.001(66.7)$ \\
\hline $17-25$ & $77(1.7)$ & $63(2.0)$ & $14(0.9)$ & \\
\hline $26-35$ & $445(9.6)$ & $349(11.2)$ & $96(6.2)$ & \\
\hline $36-45$ & $\mathrm{I} I 03(23.7)$ & $788(25.2)$ & $315(20.5)$ & \\
\hline $46-55$ & $1780(38.2)$ & $1154(37.0)$ & $626(40.7)$ & \\
\hline $56-64$ & $1255(26.9)$ & $768(24.6)$ & $487(31.7)$ & \\
\hline Mean (SD) & & $47.6(9.8)$ & $50.0(8.9)$ & $<0.001(8.10)$ \\
\hline Health plan type, n (\%) & & & & $0.005(20.4)$ \\
\hline PPO & $284 \mid(6 \mid .0)$ & $1916(62.5)$ & $925(61.5)$ & \\
\hline HMO & $686(14.7)$ & $462(15.1)$ & $224(14.9)$ & \\
\hline POS & $670(14.4)$ & $454(14.8)$ & $216(14.4)$ & \\
\hline Comprehensive & $186(4.0)$ & $106(3.5)$ & $80(5.3)$ & \\
\hline $\mathrm{CDHP}$ & $98(2.1)$ & $60(2.0)$ & $38(2.5)$ & \\
\hline Unknown & $91(2.0)$ & $56(1.8)$ & $35(2.3)$ & \\
\hline EPO & $40(0.9)$ & $32(1.0)$ & $8(0.5)$ & \\
\hline HDHP & $34(0.7)$ & $29(1.0)$ & $5(0.3)$ & \\
\hline POS with capitation & $14(0.3)$ & $7(0.2)$ & $7(0.5)$ & \\
\hline US geographic region, n (\%) & & & & $0.006(14.5)$ \\
\hline South & $2266(48.6)$ & I553 (49.7) & $713(46.4)$ & \\
\hline North Central & $1319(28.3)$ & $843(27.0)$ & $476(31.0)$ & \\
\hline West & $72 \mid(\mid 5.5)$ & $503(16.1)$ & $218(14.2)$ & \\
\hline Northeast & $334(7.2)$ & $208(6.7)$ & $126(8.2)$ & \\
\hline Unknown & $20(0.4)$ & $15(0.5)$ & $5(0.3)$ & \\
\hline
\end{tabular}

Abbreviations: CDHP, consumer-driven health plan; EPO, exclusive provider organization; HDHP, high-deductible health plan; HMO, health maintenance organization; MPR, medication possession ratio; POS, point-of-service; PPO, preferred provider organization; SD, standard deviation.

medical conditions observed in addition to FM were low back pain, dyslipidemia, and hypertension (Table 3). Rates of low back pain $(43.0 \%$ vs $38.0 \% ; P<0.001)$ and anxiety $(10.2 \%$ vs $7.8 \% ; P=0.01)$ were significantly higher in the low (compared with the high) duloxetine-adherence cohort. Rates of dyslipidemia (33.2\% vs $27.2 \% ; P<0.001)$, osteoarthritis (22.7\% vs $17.9 \% ; P<0.001)$, and psoriatic arthropathy ( $2.1 \%$ vs $1.2 \% ; P=0.017)$ were significantly higher in the high (compared with the low) duloxetine-adherence cohort. In the low duloxetine-adherence cohort, $>64 \%$ of patients used antidepressants and approximately $50 \%$ of patients used anticonvulsants prior to duloxetine initiation. In addition, approximately $75 \%$ of patients used narcotics and about $50 \%$ used nonsteroidal anti-inflammatory drugs (NSAIDs) in the prior 12 months. Rates of antidepressant (71.4\% vs $64.4 \%$; $P<0.001)$, anticonvulsant (53.5\% vs $49.3 \% ; P=0.006)$, selective serotonin reuptake inhibitor (SSRI) $(40.0 \%$ vs $30.9 \% ; P<0.001)$, pregabalin (33.0\% vs $28.7 \% ; P=0.003)$, and venlafaxine (a dual 5-HT and NE reuptake inhibitor)
(10.2\% vs $6.7 \% ; P<0.001)$ use were significantly higher in the high compared with the low duloxetine-adherence cohort.

\section{Predictors of duloxetine adherence}

Summarized in Table 4 are the factors associated with high adherence to duloxetine therapy identified using a stepwise MLR approach. Among all the variables used in the model (ie, patient demographics, comorbidities, and prior medication use), the strongest association identified was that individuals $>35$ years old were more likely to adhere to duloxetine therapy compared to patients $18-35$ years old (odds ratios $=1.42,1.80$, and 2.04 for $36-45,46-55$, and 56-64-year age-groups, respectively; all $P<0.05$ ). In addition, patients living in the North Central or Northeast regions of the US were more likely to be in the high-adherence cohort compared with the Southern region (odds ratios $=1.22$ and 1.38 for North Central and Northeast regions, respectively; both $P<0.05$ ). Other significant factors associated with high 
Table 3 Selected comorbid conditions and use of psychotropic and pain medications among patients with fibromyalgia grouped by adherence to duloxetine over prior year

\begin{tabular}{|c|c|c|c|}
\hline & $\begin{array}{l}\text { Low adherence } \\
(\text { MPR }<0.80) n(\%)\end{array}$ & $\begin{array}{l}\text { High adherence } \\
(M P R \geq 0.80) n(\%)\end{array}$ & $\begin{array}{l}P \text { values } \\
\left(\chi^{2} \text { statistics }\right)\end{array}$ \\
\hline Number of patients & 3122 & 1538 & \\
\hline \multicolumn{4}{|l|}{ Comorbid conditions } \\
\hline \multicolumn{4}{|l|}{ Pain diseases } \\
\hline Low back pain & $|34|(43.0)$ & $585(38.0)$ & $<0.001(10.3)$ \\
\hline Osteoarthritis & $560(17.9)$ & $349(22.7)$ & $<0.00 \mid(14.8)$ \\
\hline Migraine & $362(11.6)$ & $177(\mid 1.5)$ & $0.93(0.01)$ \\
\hline Rheumatoid arthritis & $220(7.1)$ & $112(7.3)$ & $0.77(0.1)$ \\
\hline Neuropathic pain other than DPNP & $226(7.2)$ & $107(7.0)$ & $0.73(0.1)$ \\
\hline Irritable bowel syndrome & $109(3.5)$ & $58(3.8)$ & $0.63(0.2)$ \\
\hline Psoriatic arthropathy & $37(1.2)$ & $32(2.1)$ & $0.017(5.7)$ \\
\hline Interstitial cystitis & $33(I .1)$ & $10(0.7)$ & $0.17(1.9)$ \\
\hline \multicolumn{4}{|l|}{ Sleep disorders } \\
\hline Sleep disorder & $511(16.4)$ & $277(18.0)$ & $0.16(2.0)$ \\
\hline \multicolumn{4}{|l|}{ Psychiatric disorders } \\
\hline Anxiety & $317(10.2)$ & $120(7.8)$ & $0.01(6.70)$ \\
\hline Bipolar disorder & $104(3.3)$ & $4 \mathrm{I}(2.7)$ & $0.22(1.5)$ \\
\hline Posttraumatic stress disorder & $24(0.8)$ & $8(0.5)$ & $0.93(0.3)$ \\
\hline \multicolumn{4}{|l|}{ Other disorders } \\
\hline Dyslipidemia & $850(27.2)$ & $511(33.2)$ & $<0.00 \mid(17.9)$ \\
\hline Hypertension & 921 (29.5) & $465(30.2)$ & $0.6 \mathrm{I}(0.3)$ \\
\hline Chronic pulmonary disease & $421(13.5)$ & $197(12.8)$ & $0.52(0.4)$ \\
\hline Hypothyroidism & $389(12.5)$ & $218(14.2)$ & $0.10(2.7)$ \\
\hline \multicolumn{4}{|l|}{ Psychotropic and pain medications } \\
\hline Anticonvulsant & $1538(49.3)$ & $823(53.5)$ & $0.006(7.4)$ \\
\hline Antidepressant & $2011(64.4)$ & 1098 (7I.4) & $<0.001(22.6)$ \\
\hline Bupropion & $290(9.3)$ & $166(10.8)$ & $0.10(2.6)$ \\
\hline COX-2 & $287(9.2)$ & $166(10.8)$ & $0.08(3.0)$ \\
\hline Dopamine agonists & $16 \mid(5.2)$ & $82(5.3)$ & $0.80(0.6)$ \\
\hline Gabapentin & $479(15.3)$ & $256(16.6)$ & $0.25(1.3)$ \\
\hline Muscle relaxants & I 496 (47.9) & $723(47.0)$ & $0.56(0.3)$ \\
\hline Narcotics & $2372(76.0)$ & II 36 (73.9) & $0.12(2.5)$ \\
\hline Benzodiazepine & $1010(32.4)$ & $484(31.5)$ & $0.54(0.4)$ \\
\hline Non-narcotics & $167(5.4)$ & $93(6.1)$ & $0.33(\mathrm{I} .0)$ \\
\hline NSAIDs & 1579 (50.6) & $774(50.3)$ & $0.87(0.03)$ \\
\hline Pregabalin & $896(28.7)$ & $507(33.0)$ & $0.003(8.9)$ \\
\hline SSRIs & $963(30.9)$ & $615(40.0)$ & $<0.001(38.5)$ \\
\hline TCAs & $499(16.0)$ & $249(16.2)$ & $0.86(0.03)$ \\
\hline Venlafaxine & $210(6.7)$ & $157(10.2)$ & $<0.00 \mid(I 7.2)$ \\
\hline
\end{tabular}

Abbreviations: COX-2, cyclooxygenase-2 selective inhibitors; DPNP, diabetic peripheral neuropathic pain; MPR, medication possession ratio; NSAIDs, nonsteroidal antiinflammatory drugs; SSRIs, selective serotonin reuptake inhibitors; TCAs, tricyclic antidepressants.

adherence to duloxetine therapy included dyslipidemia and osteoarthritis (odds ratios $=1.19$ and 1.21, respectively; both $P<0.05)$. Finally, prior use of pregabalin, antidepressants, SSRIs, or venlafaxine was associated with high duloxetine adherence (odds ratios $=1.23,1.27,1.32$, and 1.39 , respectively; all $P<0.05)$.

\section{Predictors of duloxetine persistence}

Summarized in Table 4 are the factors associated with high persistence with duloxetine therapy, identified using MLR. As with the comparable analysis for adherence, the strongest association identified was that individuals $>35$ years old were more likely to persist with duloxetine therapy compared with patients $18-35$ years old (odds ratios $=1.43,1.85$, and 1.89 for 36-45, 46-55, and 56-64-year age-groups, respectively; all $P<0.05$ ). Geographically, patients living in the North Central region of the US were more likely to be in the highpersistence cohort compared with the Southern region reference group (odds ratio $=1.18 ; P<0.05$ ). Hypothyroidism was a significant factor associated with high persistence with duloxetine therapy (odds ratio $=1.24 ; P<0.05$ ), whereas chronic pulmonary disease and interstitial cystitis were 
Table 4 Demographic and pretreatment clinical predictors of patient cohorts with fibromyalgia: multiple logistic regression

\begin{tabular}{|c|c|c|c|c|}
\hline \multirow[t]{2}{*}{ Variable } & \multicolumn{2}{|c|}{$\begin{array}{l}\text { Adherence }^{a} \\
\text { High vs low }\end{array}$} & \multicolumn{2}{|c|}{$\begin{array}{l}\text { Persistence }^{\text {b }} \\
\text { High vs low }\end{array}$} \\
\hline & $\begin{array}{l}\text { High } \\
\text { Odds } \\
\text { ratio }^{c}\end{array}$ & $\begin{array}{l}\text { low } \\
95 \% \\
\text { confidence } \\
\text { interval }\end{array}$ & $\begin{array}{l}\text { High v } \\
\text { Odds } \\
\text { ratioc }^{c}\end{array}$ & $\begin{array}{l}\text { low } \\
95 \% \\
\text { confidence } \\
\text { interval }\end{array}$ \\
\hline \multicolumn{5}{|l|}{ Age, years } \\
\hline $36-45$ vs $18-35$ & 1.42 & I.08-I.85 & 1.43 & I.I0-I.87 \\
\hline $46-55$ vs $18-35$ & 1.80 & I.39-2.33 & 1.85 & $1.44-2.38$ \\
\hline $56-64$ vs $18-35$ & 2.04 & $1.56-2.67$ & 1.89 & $1.46-2.45$ \\
\hline \multicolumn{5}{|l|}{ Geographic region } \\
\hline West vs South & 0.98 & $0.81-1.18$ & 0.90 & $0.75-1.09$ \\
\hline $\begin{array}{l}\text { North Central vs } \\
\text { South }\end{array}$ & 1.22 & I.05-I.4I & 1.18 & $\mathrm{I} .0 \mathrm{I}-\mathrm{I} .36$ \\
\hline Northeast vs South & 1.38 & I.08-I.78 & 1.03 & $0.79-1.33$ \\
\hline \multicolumn{5}{|l|}{ Prior medication use } \\
\hline Anticonvulsants & - & - & 1.36 & $1.18-1.58$ \\
\hline Antidepressants & 1.27 & I.05-I.53 & 1.27 & I.05-I.53 \\
\hline cox-2 & - & - & 1.26 & $1.03-1.55$ \\
\hline Narcotics & - & - & 0.86 & $0.74-1.00$ \\
\hline Pregabalin & 1.23 & I.07-I.42 & - & - \\
\hline SSRIs & 1.32 & I.II-I.56 & 1.28 & $|.08-| .5 \mid$ \\
\hline Venlafaxine & 1.39 & I.09-1.77 & 1.51 & $1.19-1.92$ \\
\hline \multicolumn{5}{|l|}{ Comorbidity } \\
\hline $\begin{array}{l}\text { Chronic pulmonary } \\
\text { disease }\end{array}$ & - & - & 0.79 & $0.65-0.96$ \\
\hline Dyslipidemia & 1.19 & I.03-1.37 & - & - \\
\hline Hypothyroidism & - & - & 1.24 & I.03-I.49 \\
\hline Interstitial cystitis & - & - & 0.33 & $0.14-0.80$ \\
\hline Osteoarthritis & 1.21 & I.03-1.42 & - & - \\
\hline Low back pain & 0.80 & $0.70-0.92$ & - & - \\
\hline
\end{tabular}

Notes: ${ }^{a}$ High adherence was defined as medication possession ratio (MPR) $\geq 0.80$; bHigh persistence was defined as duration of therapy $\geq 180$ days, antidepressants include SSRIs and venlafaxine; 'odds ratio $>1.0$ indicates the factor is associated with greater adherence or persistence.

Abbreviations: COX-2, cyclooxygenase-2 selective inhibitors; SSRIs, selective serotonin reuptake inhibitors.

associated with low persistence with duloxetine therapy (odds ratios $=0.79$ and 0.33 , respectively; both $P<0.05$ ). Finally, prior use of SSRIs, venlafaxine, cyclooxygenase-2 selective inhibitors, anticonvulsants, or antidepressants was associated with high persistence with duloxetine therapy (odds ratios $=1.28,1.51,1.26,1.36$, and 1.27 , respectively; all $P<0.05$ ), whereas prior use of narcotics was associated with low persistence with duloxetine therapy (odds ratio $=0.86$; $P<0.05)$.

\section{Examination of subgroups using CART analysis}

Across the whole FM patient cohort, the average MPR was 0.53. The CART model identified prior use of antidepressants, patient age, and history of osteoarthritis as the most important factors defining the high-MPR subgroups. Patients who used antidepressants, were 45 years of age or older, and had osteoarthritis were more likely to possess duloxetine (Figure 1A). The subgroup meeting these criteria accounted for 515 (11.1\%) of the 4660 patients with an elevated MPR (0.62).

Highly adherent patients accounted for $33 \%$ of the whole FM patient cohort. The CART model identified patient age and prior use of SSRIs as the most important factors defining duloxetine-adherent subgroups in patients with FM (Figure 1B). Patients who were 46.5 years of age or older and used SSRIs were more likely to adhere to duloxetine. The subgroup meeting these criteria accounted for 1011 $(21.7 \%)$ of the 4660 patients with an elevated adherence rate $(44 \%)$.

On average, the duration of therapy was 143 days across the whole FM patient cohort. The CART model identified prior use of SSRIs or venlafaxine, patient age, and use of anticonvulsants as the most important factors defining subgroups with longer duration of therapy. Patients who used SSRIs and anticonvulsants and were 44.5 years of age or older were more likely to persist with duloxetine treatment (Figure 1C). The subgroup of 621 patients (13.3\%) meeting these criteria had an average duration of therapy of 181 days. Similarly, patients who did not use SSRIs, but used venlafaxine, were also more likely to persist with duloxetine treatment (Figure 1C). The 283 patients $(6.1 \%)$ who met these criteria had an average duration of therapy of 187 days.

\section{Discussion}

The present retrospective database study analyzed a large US-based commercial claim and encounters database to examine the predictors of adherence to and persistence with duloxetine therapy in FM patients over a 1-year period. To our knowledge, this is the first study to examine persistence with duloxetine therapy or its predictors. Overall, this cohort of FM patients had a combined average duration of therapy of 279.4 days. Comparing patients with high and low persistence using multiple logistic regression, higher persistence was associated with age $>35$ years, geographic region, and prior use of antidepressants, anticonvulsant, cyclooxygenase-2 inhibitors, SSRIs, or venlafaxine. Greater age, geographic region, and prior use of antidepressants, and SSRIs or venlafaxine in particular, were also significantly associated with high adherence. Using CART analysis, which automatically detects key interactions between covariates, we independently identified prior use of SSRIs or venlafaxine and patient age as the most important predictors of 
A
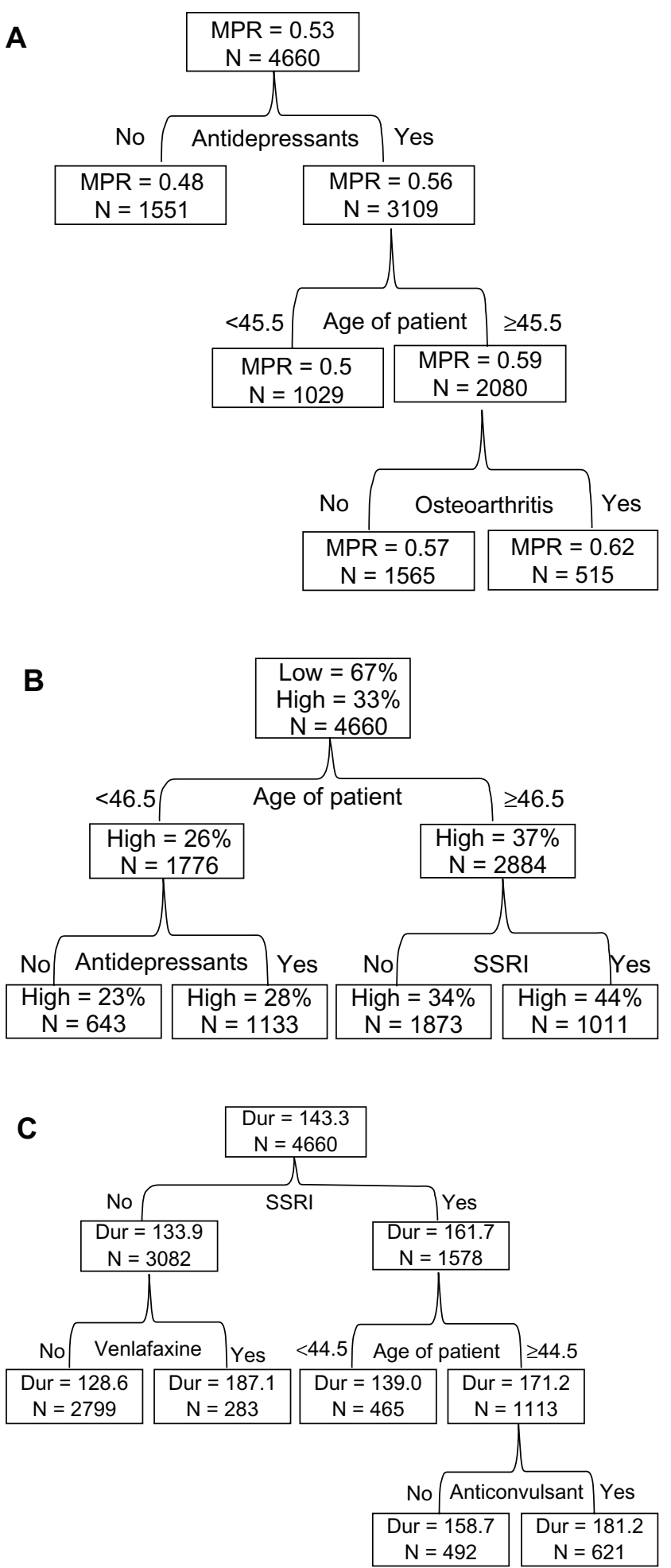

Figure I (A-C) Classification and regression tree analysis for subgroups of adherent and persistent patients with fibromyalgia. (A) Medication possession ratio (MPR): total duloxetine supply days/365. Node size $(\mathrm{N})>250$. Partition testing: F-test, $\alpha=0.05$. (B) Adherence (low, high): adherence $=$ high if $M P R \geq 0.80$; adherence $=$ low if MPR $<0.80$. Node size $(\mathrm{N})>250$. Split testing: Pearson $\chi^{2}, \alpha=0.05$. (C) Duration of therapy (Dur): duration of therapy from the index date to the earliest of either the ending date of the last prescription, the date of the first gap of $>15$ days between prescriptions, or the end of the study period (I2 months). Node size $(\mathrm{N})>250$. Partition testing: F-test, $\alpha=0.05$. persistence with duloxetine therapy (ie, longer duration of therapy) in this cohort of FM patients.

With respect to adherence to duloxetine treatment, using a criterion of MPR $\geq 0.80$, approximately $33 \%$ of the FM patients included in this study were classified as having high adherence. Predictors of high adherence to duloxetine treatment included a prior history of antidepressant use and greater age. These findings were consistent irrespective of the statistical analysis method employed (ie, MLR or CART analyses). It is interesting to note, however, that had a 6-month follow-up period been employed instead of a 1-year follow-up period, MPRs would have been considerably different across the FM patients (data not shown), which could potentially impact the factors identified as well as the predictive value of those factors. Therefore, the duration of periods assessed should be considered when comparing results across different analyses of adherence or persistence, and longer periods may be important to consider when assessing treatments for chronic conditions such as FM.

Similar to a previous study, ${ }^{5}$ approximately $90 \%$ of the FM patients were female, and most of the FM patients had many comorbid medical conditions. In general, the two adherence cohorts had similar medical history profiles; however, there were some differences with respect to prevalence: rates of anxiety and low back pain were significantly higher in the low duloxetine-adherence cohort, whereas rates of osteoarthritis, psoriatic arthropathy, dyslipidemia, and hypertension were significantly higher in the high duloxetine-adherence cohort. Given that osteoarthritis, dyslipidemia, and hypertension may be expected to be more common in older patients, it is perhaps not surprising that these were associated with the older, more adherent cohort. Notably, however, these comorbidities did not significantly associate with high persistence. This finding suggests that the relationship between comorbid disease and persistence may be different from that between comorbid disease and adherence.

As previously mentioned, FM is a complex medical condition, with patients often taking multiple medications concomitantly. ${ }^{19,20}$ Similar trends were observed in the present study, with the vast majority of FM patients taking numerous medications in the year prior to study initiation, with narcotics, antidepressants, and anticonvulsants among the most prevalent. The concomitant use of narcotics and antidepressants was common in the FM patients and concordant with the literature. ${ }^{5}$ Although not unexpected, opioid use among the FM patients was frequent despite clinical guidelines suggesting that this drug class (other than tramadol) 
should be prescribed only after other alternatives have been fully exhausted. ${ }^{23}$ In addition, although concomitant medication use was not systematically analyzed, the possibility of combined duloxetine and pregabalin therapy has been proposed previously. ${ }^{24}$ In a post hoc analysis of our data, at least one pregabalin prescription during the 1-year study period was recorded for $27 \%$ of patients with low adherence and for $30 \%$ of patients with high adherence, suggesting little or no correlation with adherence to duloxetine. Nonetheless, an in-depth analysis of concomitant medication usage could be a valuable addition to future studies.

Almost half of FM patients also used NSAIDs despite their lack of efficacy in treating FM, ${ }^{25-27}$ although this may be underestimated given that over-the-counter medication use was not captured. The widespread use of NSAIDs in this patient population may reflect the management of other painrelated comorbidities, such as low back pain and arthritis. Future studies assessing the use of NSAIDs in this patient population is certainly warranted.

This study has several limitations related to being a retrospective study based on a claims database. First, as a consequence of the study design, findings can only be interpreted as association and not causality. Second, as this was a claims database analysis, there exists potential for selection bias, miscoding of information, and consequent biases in estimation. Third, all medical conditions were identified solely based on ICD-9-CM diagnosis codes recorded in the administrative claims database, which may be subject to inaccuracies as no validation with patient medical records was performed. Fourth, prescription records were used to quantify patient adherence to therapy, which may not adequately reflect actual consumption. Finally, an important limitation was that no data pertaining to severity or duration of FM, pain levels, or socioeconomic data on education, ethnicity, employment status, income, and family environment were available. Such variables may be associated with adherence and persistence outcomes, and their inclusion might have resulted in improved identification of key patient subgroups. Thus, caution must be used when interpreting these results, particularly for some of the general factors associated with adherence and persistence, such as geographic region, which might reflect identifiable social or demographic factors that were not captured in the data sets analyzed here. Given the difficulty of reliably assessing pain levels in the absence of validated rating instruments, as well as the difficulty in accurately assessing time of onset for FM, data on the impact of pain levels and duration of FM from prospective studies may be needed to understand the impact of these factors on adherence and persistence. If such data were strongly associated with adherence or persistence, their availability would be expected to allow greater specificity in identifying those patients most likely to continue and benefit from further therapy.

In conclusion, the findings from this study indicate that one-third of FM patients were highly adherent to duloxetine therapy over a 1-year period. Patients who achieved high adherence to duloxetine therapy were significantly older and had a prior history of antidepressant use. In addition, in this cohort of FM patients, the CART model identified prior use of SSRIs or venlafaxine and patient age as the most important predictors of longer duloxetine therapy. These findings may be of use in identifying patient subgroups most likely to adhere to and persist with treatment of their FM with duloxetine, thus resulting in reduced overall health-care costs and burden for these patients and their health-care providers.

\section{Acknowledgments}

The authors would like to thank Yi Chen and Yun Fang from inVentiv Clinical Services for helping with the preparation of the data sets and data analysis, respectively. We are also grateful to Steve Able, Baojing Zhu, and Emily Zhu from Eli Lilly for reviewing this manuscript. This study was funded by Eli Lilly and Company.

\section{Disclosure}

Drs. Cui, Zhao, Novick, and Faries are full-time employees of Eli Lilly and Company and are minor stockholders of Eli Lilly and Company.This project was sponsored by Lilly Research Laboratories, Eli Lilly and Company, Indianapolis, IN, USA. Employees of Lilly were involved in the study design, analysis of data, interpretation of data, writing of the manuscript, and decision to submit the manuscript for publication.

\section{References}

1. Wright CL, Mist SD, Ross RL, Jones KD. Duloxetine for the treatment of fibromyalgia. Expert Rev Clin Immunol. 2010;6(5):745-756.

2. Schmidt-Wilcke T, Clauw DJ. Pharmacotherapy in fibromyalgia (FM) - implications for the underlying pathophysiology. Pharmacol Ther. 2010;127(3):283-294.

3. Berger A, Dukes E, Martin S, Edelsberg J, Oster G. Characteristics and healthcare costs of patients with fibromyalgia syndrome. Int J Clin Pract. 2007;61(9):1498-1508.

4. Neumann L, Buskila D. Epidemiology of fibromyalgia. Curr Pain Headache Rep. 2003;7(5):362-368.

5. Navarro RP. Contemporary management strategies for fibromyalgia. $\mathrm{Am}$ J Manag Care. 2009;15(Suppl 7):S197-S218.

6. Annemans L, Le Lay K, Taieb C. Societal and patient burden of fibromyalgia syndrome. Pharmacoeconomics. 2009;27(7): 547-559.

7. Staud R, Vierck CJ, Cannon RL, Mauderli AP, Price DD. Abnormal sensitization and temporal summation of second pain (wind-up) in patients with fibromyalgia syndrome. Pain. 2001;91(1-2):165-175. 
8. Basbaum AI, Fields HL. Endogenous pain control systems: brainstem spinal pathways and endorphin circuitry. Annu Rev Neurosci. 1984;7: 309-338.

9. Bannister K, Bee LA, Dickenson AH. Preclinical and early clinical investigations related to monoaminergic pain modulation. Neurotherapeutics. 2009;6(4):703-712.

10. Bellingham GA, Peng PW. Duloxetine: a review of its pharmacology and use in chronic pain management. Reg Anesth Pain Med. 2010;35(3): 294-303.

11. Legangneux E, Mora JJ, Spreux-Varoquaux O, et al. Cerebrospinal fluid biogenic amine metabolites, plasma-rich platelet serotonin and $[3 \mathrm{H}]$ imipramine reuptake in the primary fibromyalgia syndrome. Rheumatology (Oxford). 2001;40(3):290-296.

12. Russell IJ, Vaeroy H, Javors M, Nyberg F. Cerebrospinal fluid biogenic amine metabolites in fibromyalgia/fibrositis syndrome and rheumatoid arthritis. Arthritis Rheum. 1992;35(5):550-556.

13. Arnold LM, Rosen A, Pritchett YL, et al. A randomized, double-blind, placebo-controlled trial of duloxetine in the treatment of women with fibromyalgia with or without major depressive disorder. Pain. 2005;119(1-3):5-15.

14. Arnold LM, Lu Y, Crofford LJ, et al. A double-blind, multicenter trial comparing duloxetine with placebo in the treatment of fibromyalgia patients with or without major depressive disorder. Arthritis Rheum. 2004;50(9):2974-2984.

15. Russell IJ, Mease PJ, Smith TR, et al. Efficacy and safety of duloxetine for treatment of fibromyalgia in patients with or without major depressive disorder: results from a 6-month, randomized, double-blind placebo-controlled, fixed-dose trial. Pain. 2008;136(3):432-444.

16. Chappell AS, Bradley LA, Wiltse C, Detke MJ, D'Souza DN, Spaeth M. A six-month double-blind, placebo-controlled, randomized clinical trial of duloxetine for the treatment of fibromyalgia. Int J Gen Med. 2009;1:91-102.

17. Kleinman NL, Sanchez RJ, Lynch WD, Cappelleri JC, Beren IA, Joshi AV. Health outcomes and costs among employees with fibromyalgia treated with pregabalin vs standard of care. Pain Pract. 2011;11(6): $540-551$
18. Zhao Y, Sun P, Watson P, Mitchell B, Swindle R. Comparison of medication adherence and healthcare costs between duloxetine and pregabalin initiators among patients with fibromyalgia. Pain Pract. 2011;11(3):204-216.

19. Zhao Y, Chen SY, Wu N, Fraser KA, Boulanger L. Medication adherence and healthcare costs among fibromyalgia patients treated with duloxetine. Pain Pract. 2011;11(4):381-391.

20. Wu N, Chen S, Boulanger L, Rao P, Zhao Y. Average daily dose, medication adherence, and healthcare costs among commerciallyinsured patients with fibromyalgia treated with duloxetine. Curr Med Res Opin. 2011;27(6):1131-1139.

21. Cramer JA, Roy A, Burrell A, et al. Medication compliance and persistence: terminology and definitions. Value Health. 2008;11(1): 44- 47.

22. American Psychiatric Association. Practice Guideline for Treatment of Patients with Major Depressive Disorder, 2nd ed. Washington: American Psychiatric Association Press; 2000.

23. Fitzcharles MA, Ste-Marie PA, Gamsa A, Ware MA, Shir Y. Opioid use, misuse, and abuse in patients labeled as fibromyalgia. Am J Med. 2011;124(10):955-960.

24. Serra E. Duloxetine and pregabalin: safe and effective for the long-term treatment of fibromyalgia? Nat Clin Pract Neurol. 2008;4:594-595.

25. Mease P. Fibromyalgia syndrome: review of clinical presentation, pathogenesis, outcome measures, and treatment. J Rheumatol Suppl. 2005;75:6-21.

26. Yunus MB, Masi AT, Aldag JC. Short term effects of ibuprofen in primary fibromyalgia syndrome: a double blind, placebo controlled trial. J Rheumatol. 1989;16(4):527-532.

27. Solitar BM. Fibromyalgia: knowns, unknowns, and current treatment Bull NYU Hosp Jt Dis. 2010;68(3):157-161.
Journal of Pain Research

\section{Publish your work in this journal}

The Journal of Pain Research is an international, peer-reviewed, open access, online journal that welcomes laboratory and clinical findings in the fields of pain research and the prevention and management of pain. Original research, reviews, symposium reports, hypothesis formation and commentaries are all considered for publication.

\section{Dovepress}

The manuscript management system is completely online and includes a very quick and fair peer-review system, which is all easy to use. Visit http://www.dovepress.com/testimonials.php to read real quotes from published authors. 